\title{
LETTER \\ Improved Orthogonal Fractal Super-Resolution Using Range Adjustment and Domain Extension
}

\author{
Moojae $\mathrm{LEE}^{\dagger \mathrm{a})}$, Nonmember, Jung-Ju $\mathrm{CHOI}^{\dagger \dagger b)}$, Member, and Youngcheul $\mathrm{WEE}^{\dagger c)}$, Nonmember
}

\begin{abstract}
SUMMARY This paper presents a modified orthogonal fractal superresolution (OFSR) method to improve the visual quality of an image along sharp edges. Although the OFSR method constructs a high-quality highresolution image from a low-resolution counterpart, there are ringing artifacts observed along sharp edges which make the visual quality relatively low with respect to the numerical quantity. These artifacts are mainly caused by unnecessarily exaggerated pixel contrast along sharp edges within a range block. We restrict each contracted pixel value in a range block to a value between the minimum and maximum of its domain block pixel values. We also extend the domain block of the contraction function and find a better domain block using the range block mean. At the final step of the iteration, we adjust each pixel in the range block so that the range block mean and the corresponding pixel value of the lowresolution image are equal. According to our experimental results, the proposed method improves the visual quality along sharp edges and shows higher levels of numerical quantity than the OFSR method.

key words: super-resolution, orthogonal fractal coding
\end{abstract}

\section{Introduction}

We present an improved orthogonal fractal super-resolution method to construct an enhanced high-resolution (HR) image from a single low-resolution (LR) counterpart. Due to the high demand for HR images in hand-held consumer electronics such as a smartphone with a built-in high-quality camera, the ability to capture, store, and transmit HR images is now a desired feature of these devices. Hence, the super-resolution technique has become attractive as an efficient technique for producing high-quality HR images from stored and/or transmitted LR images.

There are many super-resolution techniques that create HR images from multiple LR images using various motion estimation methods. Callicó et al. [1] presented a very useful survey which compared the quality and performance of various methods. There are also many super-resolution techniques that work with a single counterpart image, including a filter-based method [2], an example-based method [3], [4], an edge-based method [5]-[8], and a reconstructionbased method [9]. Recently, Wee and Shin [10] introduced an orthogonal fractal super-resolution (the OFSR, hereafter)

Manuscript received September 19, 2012.

Manuscript revised February 26, 2013.

${ }^{\dagger}$ The authors are with the Department of Information and Computer Engineering, Ajou University, Suwon 443-749, Rep. of Korea.

${ }^{\dagger \dagger}$ The author is with the Department of Digital Media, Ajou University, Suwon 443-749, Rep. of Korea.

a) E-mail: moojae@ajou.ac.kr

b) E-mail: jungju@ajou.ac.kr

c) E-mail: ycwee@ajou.ac.kr

DOI: 10.1587/transinf.E96.D.1890 method that provides a fixed domain block to find selfsimilarity from a given range block and a fixed scaling factor with a suggested contraction function.

Assuming that an LR image consists of range block means of size $m \times n$, the OFSR constructs an HR image $2 m \times 2 n$ in size at each iteration step $k$ such that

$$
R_{i}^{(k)}=\bar{R}_{i} U+s \mu\left(D_{i}^{(k-1)}-\bar{D}_{i}^{(k-1)} U\right),
$$

where $R_{i}$ is a $2 \times 2$ range block, $D_{i}$ is a $4 \times 4$ domain block corresponding to the range block, $\bar{D}_{i}$ is the mean of $D_{i}, \mu$ is a contraction function, $s$ is a fixed scale factor, and $U$ is a $2 \times 2$ matrix whose entries are all one. They proposed that the domain block $D$ includes the range block $R$ at its center. The domain block $D$ is contracted into a $2 \times 2$ contracted block $B$ by means of the pixel-by-pixel contraction function $\mu$ and the scaling factor $s$ is set to 0.75 (see [10] for more details).

Although the OFSR algorithm can construct an HR image efficiently with high levels of numerical quantity, its primary drawback is the visual quality along sharp edges with respect to the numerical quantity [10]. There are ringing artifacts observed along the sharp edges, where the OFSR method unnecessarily exaggerates the pixel contrast. In this paper, we propose an improved OFSR method that shows better visual quality along the sharp edges as well as better numerical quantity. The remainder of this paper is organized as follows. Section 2 describes the improved OFSR method by means of a new contraction function, range adjustment, and domain extension techniques. Our experimental results are presented in Sect. 3 and the conclusion follows in Sect. 4.

\section{Improved Orthogonal Fractal Super-Resolution}

\subsection{New Contraction Function}

In the OFSR method [10], the $4 \times 4$ domain $D$ is contracted into the $2 \times 2$ block $B=\left[\begin{array}{ll}b_{0} & b_{1} \\ b_{2} & b_{3}\end{array}\right]$ using the contraction function $\mu$ such that (see Fig. 1)

$$
\begin{aligned}
& b_{0}=d_{5}^{\prime}=w_{1} d_{5}+w_{2} d_{1}+w_{2} d_{4}+w_{3} d_{0}, \\
& b_{1}=d_{6}^{\prime}=w_{1} d_{6}+w_{2} d_{2}+w_{2} d_{7}+w_{3} d_{3}, \\
& b_{2}=d_{9}^{\prime}=w_{1} d_{9}+w_{2} d_{8}+w_{2} d_{13}+w_{3} d_{12}, \\
& b_{3}=d_{10}^{\prime}=w_{1} d_{10}+w_{2} d_{11}+w_{2} d_{14}+w_{3} d_{15} .
\end{aligned}
$$

Because the OFSR method considers only the horizontal and vertical pixels in $D$ with respect to the corresponding 

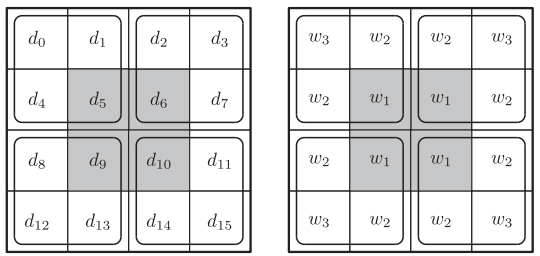

Fig. 1 A $4 \times 4$ domain block $D$ (left) and its weight kernel (right). The corresponding $2 \times 2$ range block $R$ is denoted in grey.

range block $R$ but not diagonal pixels, the weights $w_{1}, w_{2}$, and $w_{3}$ for the contraction function are $0.5,0.25$, and 0.0 , respectively. Considering only horizontal and vertical neighboring pixels may not represent the diagonal edges effectively in the reconstructed HR image. Through extensive experiments with various standard images, we empirically determined an improved weight kernel for a better contraction function with $w_{1}=0.45, w_{2}=0.3175$, and $w_{3}=-0.085$. The negative weight of $w_{3}$ implies that the weight of the diagonal pixel is already incorporated partially with its horizontal and vertical weights $w_{2}$.

\subsection{Range Adjustment}

\subsubsection{Min-Max Adjustment}

Because the pixel values of the range block can be computed from the pixel values of the domain block iteratively, any cumulative error that arises during the iterations may be repetitively enlarged. When a diagonal sharp edge passes through the domain block, the OFSR method makes dark pixels darker and light pixels lighter with each iteration step. In this case, the OFSR algorithm exaggerates the contrast unnecessarily around the sharp edges, which eventually causes ringing artifacts along the sharp edges. To reduce these artifacts, we restrict the pixel values of the contracted block $B$ between the minimum and maximum values within a specified kernel that consists of $2 \times 2$ pixels in the range block as well as 4 horizontal pixels and 4 vertical pixels in the corresponding domain block. Because this restriction prevents the reconstructed range block pixel values from being greater than the maximum or from being less than the minimum in the kernel, the ringing artifacts along the diagonal sharp edges are effectively reduced.

\subsubsection{Mean Adjustment}

The orthogonal fractal coding is mean-invariant, as shown in Eq. (1). For a pixel $\bar{r}_{i}$ in the LR image, the OFSR method generates a $2 \times 2$ range block $R_{i}^{(k)}$ at each iteration step $k$, where the mean of the range block $R_{i}^{(k)}$ is used as the value of $\bar{r}_{i}$ in the next iteration step. Note that $\bar{r}_{i}$ remains the same during the iterations. However, due to error accumulation that arises during the iterations, the cumulative error $e_{i}$ between $\bar{R}_{i}^{(k)}$ and $\bar{r}_{i}$ increases. To make the value of $\bar{R}_{i}$ in the HR image equal to that of $\bar{r}_{i}$ in the LR image, we adjust the range block $R_{i}$ in the HR image by adding $\bar{e}_{i}=e_{i} / 4$ to

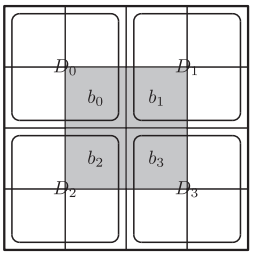

(a) $\mu_{1}$

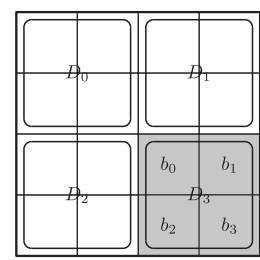

(b) $\mu_{2}$

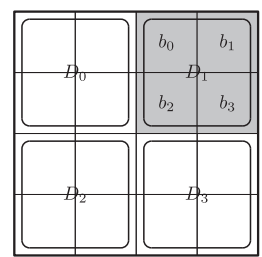

(d) $\mu_{4}$

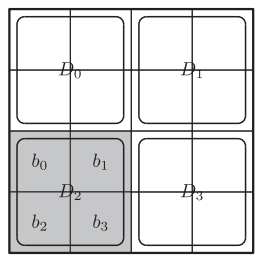

(c) $\mu_{3}$

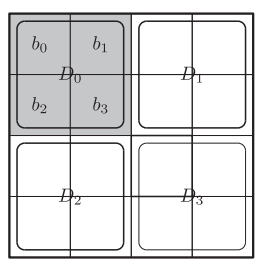

(e) $\mu_{5}$
Fig. 2 Extended domain blocks and their contraction functions.

each pixel of the range block, which achieves a better PSNR (Peak Signal-to-Noise Ratio) in the HR image.

The proposed mean adjustment technique for the OFSR method is somewhat analogous to back-projection techniques [11] to minimize the reconstruction error with various iterative procedures [9], [12], [13]. One of the important things in back-projection techniques is to design an efficient back-projection kernel. Because the OFSR is both naturally iterative and mean-invariant, the back-projection kernel of the OFSR is a uniform kernel over the contracted range block during every iteration step. However, when applying the mean adjustment technique to the OFSR repeatedly, the reconstructed image becomes blurry. We found that applying the mean adjustment technique at the time of the last iteration sufficiently improves the visual quality while preserving the sharpness as well.

\subsection{Domain Extension}

One of the advantages of the OFSR method is that it offers fast computation through its selection of a fixed domain block, with which good self-similarity is achieved. Although selecting a $4 \times 4$ domain block including the range block at its center can lead to excellent performance, we can extend this concept to the selection of a $4 \times 4$ domain block that includes the range block but not necessarily at its center in an effort to find better self-similarity in distant domain blocks.

Let $\mu_{1}$ be a contraction function over the domain block that includes the range block at its center, as in the OFSR, and let $\mu_{2}, \mu_{3}, \mu_{4}$, and $\mu_{5}$ be the contract functions over the domain blocks that include the range block at their lower right, lower left, upper right, and upper left corners, respectively (see Fig. 2). When a $4 \times 4$ domain block $D$ is contracted into a $2 \times 2$ block $B$, each pixel $b_{i}$ in $B$ has its corresponding domain sub-block according to its relative position in $B$. For example, for the contraction function $\mu_{2}$ the pixel $b_{0}$ at the upper left corner in the range block is contracted from the upper left $2 \times 2$ domain sub-block $D_{0}$ (see 
Fig. 2 (b)).

For a given range block $R$, we select the best domain block from among the five domain block configurations. Each domain block $D$ is contracted into its corresponding block $\mathrm{B}$ and the mean $\bar{b}$ of each block $B$ is then computed. The best domain block $D$ whose error between $\bar{b}$ and $\bar{r}$ is the lowest is selected. Here, $\bar{r}$ denotes the mean of the range block.

We do not apply the domain block extension technique for every iteration step $k$ in the OFSR method. Because the HR image from the first three iterations is very blocky, it is difficult to find a similar domain block from among the four distant domain blocks of Fig. 2 (b)-(e). Furthermore, because the HR image is rarely improved after three iterations in the OFSR method, we utilize our domain block extension technique only when $k>3$. We also apply the domain block extension technique at most three times, as this affects the convergence of the contracted block due to the distant domain blocks.

\section{Experimental Results}

For a quantitative comparison between the proposed method and the OFSR method [10], we applied each method to the nine $512 \times 512$ standard images shown in Table 1 . These images were down-sampled to a quarter of their original size using a $2 \times 2$ box filter and then restored into their original size using the OFSR and our proposed methods.

In the OFSR method, the quality improvement is marginal after the first three iterations and in fact may worsen as $k$ increases. Therefore, we compare the PSNR of the proposed method to that of the OFSR method when $k=3$ to evaluate the performance of the min-max adjustment technique. We apply our domain extension technique at most three times when $k>3$ and apply the mean adjustment technique at the last step. To evaluate the performance of our domain extension and the mean adjustment techniques, we also compare the PSNR of the proposed method to that of the OFSR method when both methods reach their maximum PSNR.

Table 1 shows the PSNR of the restored images to the original images when $k=3$ and when the PSNR reaches its maximum at $k_{\max }$ for each of the OFSR and our methods. The values within the parenthesis are the PSNR gains when $k=3$, which were achieved mainly by the min-max adjustment with respect to the OFSR method. The additional PSNR gains at $k_{\max }$ mainly by the domain extension technique are measured from the PSNR of the proposed method when $k=3$, not from the maximum PSNR of the OFSR. The last two images, Bike and Peppers2, were not involved to find our improved weight kernel, but applying the improved weight kernel and our proposed method to those images results in a higher PSNR than that of the OFSR. This implies that the proposed method is also effectively applicable to images other than those in this paper. As it is readily apparent in the table, the min-max adjustment technique achieves higher PSNR than does the OFSR for the first three
Table 1 PSNR of the restored images.

\begin{tabular}{lcccccc}
\hline & OFSR & Proposed & \multicolumn{2}{c}{ OFSR } & \multicolumn{2}{c}{ Proposed } \\
& at $k=3$ & at $k=3$ & max. & $k_{\max }$ & max. & $k_{\max }$ \\
\hline Airplane & 31.30 & $32.51(1.21)$ & 31.33 & 9 & $33.24(0.74)$ & 10 \\
Baboon & 23.95 & $24.10(0.15)$ & 23.95 & 2 & $24.16(0.06)$ & 10 \\
Barbara & 28.30 & $28.47(0.17)$ & 28.30 & 3 & $28.55(0.08)$ & 16 \\
Lenna & 35.58 & $35.70(0.12)$ & 35.69 & 12 & $36.03(0.33)$ & 14 \\
Peppers1 & 39.08 & $39.33(0.25)$ & 39.12 & 11 & $39.50(0.17)$ & 10 \\
Sailboat & 30.02 & $30.45(0.44)$ & 30.05 & 10 & $30.77(0.32)$ & 11 \\
Tiffany & 30.95 & $32.20(1.24)$ & 30.95 & 3 & $32.74(0.55)$ & 10 \\
Bike & 31.28 & $31.60(0.33)$ & 31.47 & 12 & $32.03(0.42)$ & 11 \\
Peppers2 & 31.61 & $32.66(1.05)$ & 31.61 & 3 & $33.16(0.50)$ & 9 \\
\hline Average & 31.34 & $31.89(0.55)$ & 31.38 & & $32.24(0.35)$ & \\
\hline
\end{tabular}

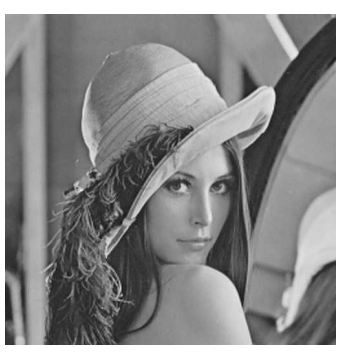

(a)

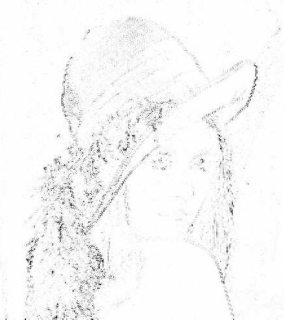

(c)

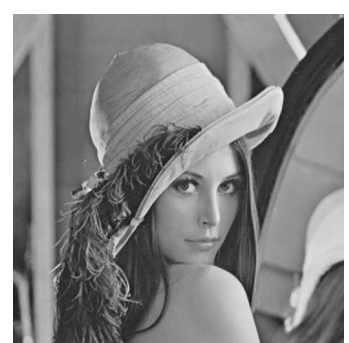

(b)
Fig. 3 Images restored by the OFSR in (a) and by the proposed method in (b) with their corresponding error images in (c) and (d), respectively.

steps. The proposed method always shows a better PSNR even when $k=3$ than the maximum PSNR of the OFSR. Furthermore, applying the domain extension and then the mean adjustment techniques improves the PSNR more, by $0.35 \mathrm{~dB}$, after the first three iterations compared to this result using the OFSR, which improves it by $0.04 \mathrm{~dB}$.

Figure 3 shows Lenna images restored by the OFSR and by the proposed method with their corresponding error images from the original image. Note that the dark pixel denotes a large degree of error in this case. It is clear that the error along the diagonal sharp edges in the proposed method is smaller than that in the OFSR. Figure 4 shows the enlarged parts of the images reconstructed by the OFSR and by the proposed method along with the corresponding parts of the error images.

Table 2 shows the performance of the proposed method compared to the conventional methods and the OFSR. We tested them on a PC with a Pentium processor $(2.53 \mathrm{GHz})$ 


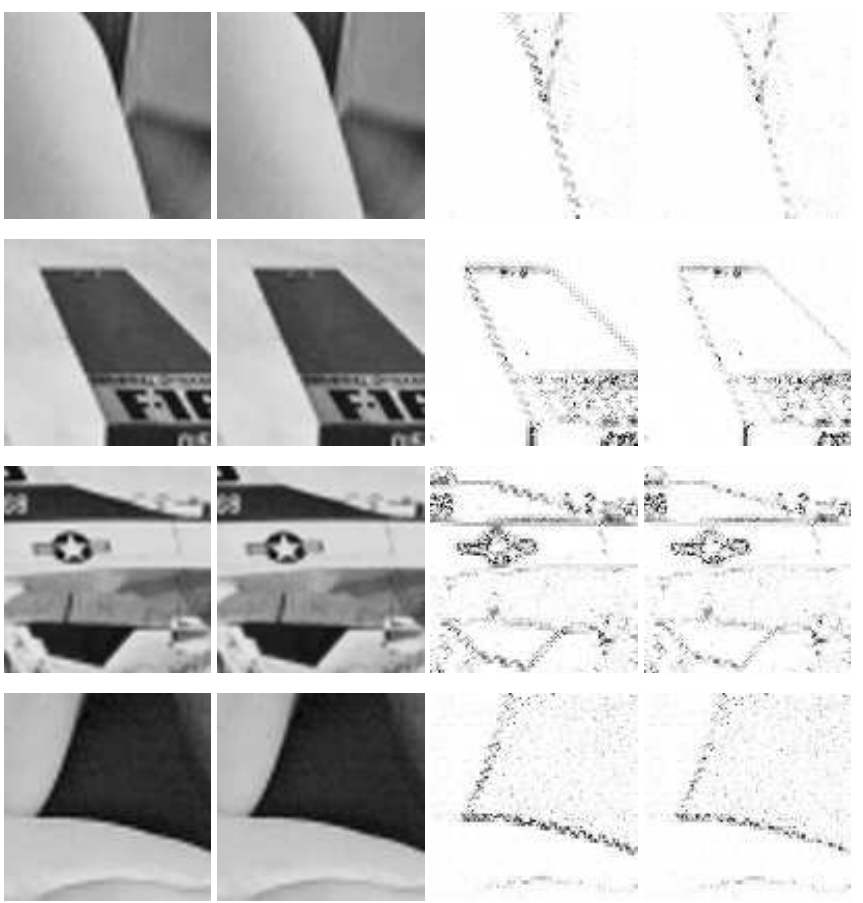

Fig. 4 Enlarged parts of images reconstructed by the OFSR (first) and by the proposed method (second) with their corresponding parts of error images (the third for the first and the fourth for the second).

Table 2 Performance comparison (Elapsed Time).

\begin{tabular}{lcccccc}
\hline \multirow{2}{*}{ Methods } & \multicolumn{5}{c}{ Time $(\mathrm{ms})$ at $k$} \\
\cline { 2 - 7 } & 1 & 2 & 3 & 4 & 5 & 6 \\
\hline OFSR & 2.6 & 5.5 & 8.3 & 11.1 & 13.7 & 16.4 \\
Proposed & 8.3 & 16.7 & 25.1 & 33.5 & 45.4 & 57.3 \\
bicubic & \multicolumn{7}{c}{52.3} \\
Lanczos-3 & \multicolumn{7}{c}{78.9} \\
\hline
\end{tabular}

and 4 GB memory. Due to our new contraction weights and mean adjustment technique, it was necessary to use floatingpoint precision computation. Because we re-implement the methods, direct comparisons with the result in [10] are likely to be meaningless. We rather compared the execution time performance by means of the average ratio of the proposed method to the OFSR. The proposed method requires a 3.18fold increase in the execution time compared to that of the OFSR method. However, the execution time of the proposed method is still comparable to or faster than the conventional methods such as bicubic and Lanczos-3 filter methods.

\section{Conclusion}

In this paper, we presented an improved OFSR method using min-max adjustment, mean adjustment, and domain extension techniques. The proposed min-max adjustment technique achieves higher PSNR than does the OFSR for the first three iterations by $0.55 \mathrm{~dB}$ on average. The domain extension and mean adjustment techniques additionally im- prove the maximum PSNR after the first three iterations by $0.35 \mathrm{~dB}$ on average. One of the drawbacks of the OFSR method is that the improvement of the visual quality does not match that of the numerical quantity, particularly along sharp edges. The proposed method effectively prevents pixel contrast from being exaggerated unnecessarily around sharp edges, which reduces the ringing artifacts along the edges. According to our experimental results, the proposed method improves the numerical quantity better than the OFSR while also showing better visual quality along sharp edges.

\section{Acknowledgment}

This research was partially supported by the National Research Foundation of Korea (NRF) grant funded by the Korean government (MEST) (No. 2012R1A1A2007264) and partially supported by the Industrial Strategic Technology Development Program (No. 10041743) funded by the Ministry of Knowledge Economy (MKE, Korea).

\section{References}

[1] G. Callico, S. Lopez, O. Sosa, J. Lopez, and R. Sarmiento, "Analysis of fast block matching motion estimation algorithms for video superresolution systems," IEEE Trans. Consum. Electron., vol.54, no.3, pp.1430-1438, 2008.

[2] H. Hou and H. Andrews, "Cubic splines for image interpolation and digital filtering," IEEE Trans. Acoust. Speech Signal Process., vol.26, no.6, pp.508-517, 1978.

[3] W. Freeman, T. Jones, and E. Pasztor, "Example-based superresolution," IEEE Comput. Graph. Appli., vol.22, no.2, pp.56-65, 2002.

[4] J. Sun, N.N. Zheng, H. Tao, and H.Y. Shum, "Image hallucination with primal sketch priors," Proc. IEEE Computer Society Conference on Computer Vision and Pattern Recognition, pp.II-729-36, 2003.

[5] K. Jensen and D. Anastassiou, "Subpixel edge localization and the interpolation of still images," IEEE Trans. Image Process., vol.4, no.3, pp.285-295, 1995.

[6] X. Li and M. Orchard, "New edge-directed interpolation," IEEE Trans. Image Process., vol.10, no.10, pp.1521-1527, 2001.

[7] L. Zhang and X. Wu, "An edge-guided image interpolation algorithm via directional filtering and data fusion," IEEE Trans. Image Process., vol.15, no.8, pp.2226-2238, 2006.

[8] M. Li and T. Nguyen, "Markov random field model-based edgedirected image interpolation," IEEE Trans. Image Process., vol.17, no.7, pp.1121-1128, 2008.

[9] S. Dai, M. Han, Y. Wu, and Y. Gong, "Bilateral back-projection for single image super resolution," Proc. IEEE International Conference on Multimedia and Expo, pp.1039-1042, 2007.

[10] Y.C. Wee and H.J. Shin, "A novel fast fractal super resolution technique,” IEEE Trans. Consum. Electron., vol.56, no.3, pp.1537-1541, 2010.

[11] M. Irani and S. Peleg, "Motion analysis for image enhancement: Resolution, occlusion, and transparency," J. Visual Communication and Image Representation, vol.4, no.4, pp.324-335, 1993.

[12] W. Dong, L. Zhang, G. Shi, and X. Wu, "Nonlocal back-projection for adaptive image enlargement," Proc. 16th IEEE International Conference on Image Processing (ICIP), pp.349-352, 2009.

[13] X. Liang and Z. Gan, "Improved non-local iterative back-projection method for image super-resolution," Proc. 6th International Conference on Image and Graphics (ICIG), pp.176-181, 2011. 